\title{
Optimal Stopping Time for Holding an Asset
}

\author{
Pham Van Khanh \\ Military Technical Academy, Hanoi, Vietnam \\ Email: van_khanh1178@yahoo.com
}

Received September 21, 2012; revised October 22, 2012; accepted November 3, 2012

\begin{abstract}
In this paper, we consider the problem to determine the optimal time to sell an asset that its price conforms to the Black-Schole model but its drift is a discrete random variable taking one of two given values and this probability distribution behavior changes chronologically. The result of finding the optimal strategy to sell the asset is the first time asset price falling into deterministic time-dependent boundary. Moreover, the boundary is represented by an increasing and continuous monotone function satisfying a nonlinear integral equation. We also conduct to find the empirical optimization boundary and simulate the asset price process.
\end{abstract}

Keywords: Optimal Stopping Time; Boundary; Brownian Motion; Black-Schole Model

\section{Introduction}

In [1], Shiryaev and Peskir have considered the problem:

$$
V_{*}=\inf _{\tau \in M}\left(W_{\tau}-\max _{0 \leq t \leq 1} W_{t}\right)^{2}
$$

where $W_{\tau}$ is standard Brownian process and they found the optimal stopping time as:

$$
\tau_{*}=\inf \left\{0 \leq t \leq 1: S_{t}-W_{t} \geq z^{*} \sqrt{1-t}\right\}
$$

where $z^{*}$ is the solution of the equation

$$
4 \Phi(z)-2 z \varphi(z)-3=0, S_{t}=\max _{0 \leq s \leq t} W_{s}, \varphi(x)=\frac{1}{\sqrt{2 \pi}} \mathrm{e}^{-x^{2} / 2}
$$

and $\Phi(x)=\frac{1}{\sqrt{2 \pi}} \int_{-\infty}^{x} \mathrm{e}^{-y^{2} / 2} \mathrm{~d} y$.

The simulation for $W_{\tau}$, boundary $z^{*} \sqrt{1-t}$ and $S_{t}-W_{t}$ are given in Figures 1-3.

In [2], Albert Shiryaev, Zouquan $\mathrm{Xu}$ and $\mathrm{Xun} \mathrm{Yu}$ Zhou solve the following problem: There is an investor holding a stock, and he needs to decide when to sell it for the last time with given time to sell $\mathrm{T}$. It is obvious that he wants to sell at a time of highest price on the interval from 0 to T. Assume that the discounted share price $X_{t}$ complies with the following dynamic equation:

$$
\mathrm{d} X_{t}=(a-r) X_{t} \mathrm{~d} t+\sigma X_{t} \mathrm{~d} W_{t}, X_{0}=1
$$

on a filtered probability space $\left(\Omega, \mathcal{F},\left\{\mathcal{F}_{t}\right\}, P\right)$ where $a$ is the growth rate of the price and $\sigma>0$ is the volatility, $r$ is interest rate, $W_{\tau}$ is the standard Brownian motion with $W_{0}=0$ under the measure $P$. Here, $\left\{\mathcal{F}_{t}\right\}_{t \geq 0}$ is Pincreasing filter generated by $W_{\tau}$. Then, $X_{t}=\mathrm{e}^{\mu t+\sigma W_{t}}$

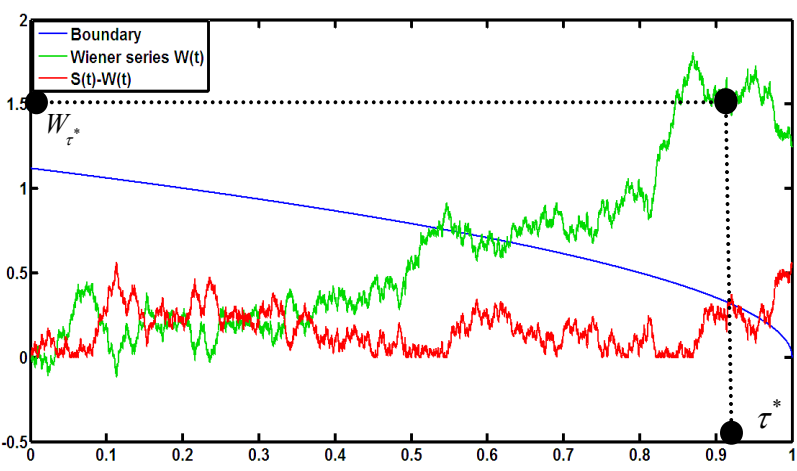

Figure 1. A simulation for stopping time in (1.2). The optimal stopping time $\tau^{*}$ is the first time the boundary line (blue line) lies below the line describes the process $S_{t}-W_{t}$ (red line). In this case $W_{\tau^{*}}$ is very large but is not $\max _{0 \leq t \leq 1} W_{t}$.

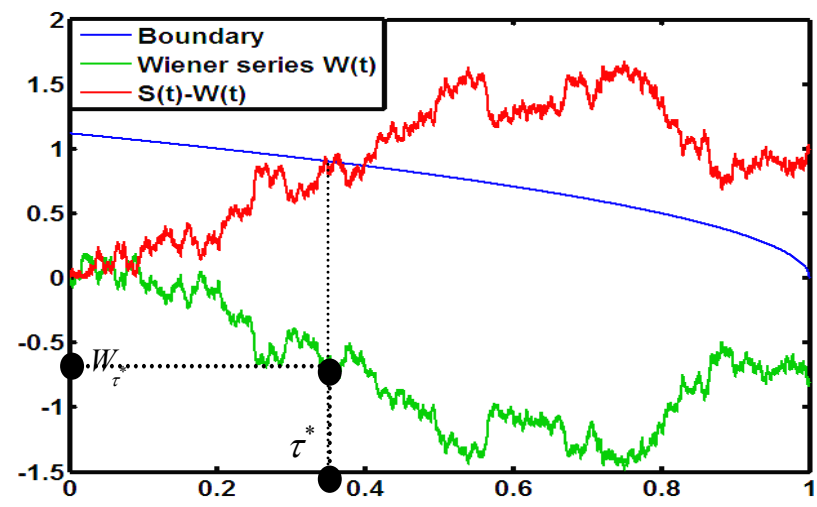

Figure 2. A simulation for the stopping time in (1.2). In this case $W_{\tau^{*}}$ is less than 0 but is not $\max _{0 \leq t \leq 1} W_{t}$. 


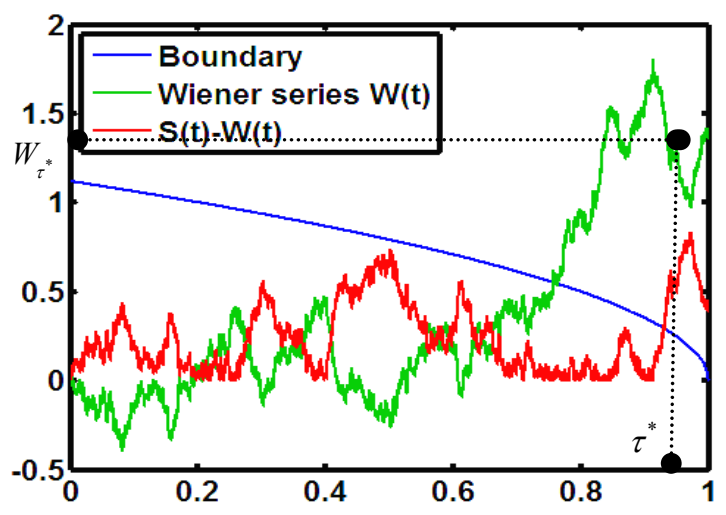

Figure 3. A simulation for the stopping time in (1.2). In this case $W_{\tau^{*}}$ is very large but is not $\max _{0 \leq t \leq 1} W_{t}$.

where

$$
\mu=a-r-\frac{1}{2} \sigma^{2} . \text { We define: } M_{t}=\max _{0 \leq s \leq t} X_{s}, \quad t \geq 0
$$

and $\alpha=\frac{a-r}{\sigma^{2}}$.

Then, the following cases:

- If $\alpha>\frac{1}{2}, \tau^{*}=T$ is an unique optimal selling time.

- If $\alpha=\frac{1}{2}, \tau^{*}=0$ or $\tau^{*}=T$ are optimal selling times.

- If $\alpha \leq 0, \tau^{*}=0$ is an unique optimal selling time.

In this paper, we will find the optimal time to sell a stock when the appreciation rate is the random variable taking one of two given values $a_{l}$ and $a_{h}$.

\section{The Problem of Finding the Optimal Selling Time}

Assume that the asset price process $X_{t}$ follows a geometric Brownian motion with its drift is a random variable taking one of two given values $a_{l}$ or $a_{h}$, the volatility $\sigma>0$ is constant, i.e.

$$
\mathrm{d} X_{t}=a_{t} X_{t} \mathrm{~d} t+\sigma X_{t} \mathrm{~d} W_{t}, \quad t \geq 0
$$

where $W_{t}$ is a standard Brownian motion independent with $a_{t}$ of the probability space $(\Omega, \mathcal{F}, P)$. Assume $(\Omega, \mathcal{F}, P)$ is a complete probability space with nondecrease $\sigma$-field. Suppose $a_{l}$ and $a_{h}$ satisfy $a_{l}<r<$ $a_{h}$, where $r$ is the interest rate and it is constant and the initial value of assets $X_{0}$ is a positive constant.

Investors holding assets need to decide when to sell it for the last time with given time to sell them is $T$. Knowing that at the initial time distribution of $\alpha$ as

$$
P\left(a=a_{h}\right)=\pi_{0} ; P\left(a=a_{\tau}\right)=1-\pi_{0}
$$

At time $t>0$ we put $\pi_{t}=P\left\{a=a_{h} \mid \mathcal{F}_{t}^{X}\right\}$, where $\left\{\mathcal{F}_{t}^{X}\right\}, t \in[0, T]$ is the completion of the filtration gen- erated by $X$.

The problem is finding $\mathcal{F}^{X}$-stopping time $\tau, 0 \leq \tau \leq T$ such that

$$
\mathrm{V}=\sup _{0 \leq \tau \leq T} E\left[\mathrm{e}^{-r \tau} X_{\tau}\right]
$$

where supremum is taken in $\mathcal{F}^{X}$-stopping time $\tau, 0 \leq \tau$ $\leq T$.

The price process $X_{t}$ and posterior probability process $\pi_{t}$ satisfying the equations

$$
\left\{\begin{array}{l}
\frac{\mathrm{d} X_{t}}{X_{t}}=\left(a_{l}+\pi_{t}\left(a_{h}-a_{l}\right)\right)+\mathrm{d} t+\sigma \mathrm{d} \bar{W}_{t} \\
\mathrm{~d} \pi_{t}=\frac{a_{h}-a_{l}}{\sigma} \pi_{t}\left(1-\pi_{t}\right) \mathrm{d} \bar{W}_{t}
\end{array}\right.
$$

where $\left(\bar{W}, \mathcal{F}^{X}\right)$ is a P-Brownian motion defined by:

$$
\bar{W}_{t}=\int_{0}^{t} \frac{\mathrm{d} X_{u}-\left[\left(1-\pi_{t}\right) a_{l}-\pi_{t} a_{h}\right] \mathrm{d} u}{\sigma X_{u}}
$$

(see [3], theorem 9.1)

Define the process $\left\{\tilde{W}_{t}\right\}$ by:

$$
\mathrm{d} \tilde{W}_{t}=\left(\omega \pi_{t}-\sigma\right) \mathrm{d} t+\mathrm{d} \bar{W}_{t}
$$

and a new measure $P^{*}$ satisfying:

$$
\begin{aligned}
\frac{\mathrm{d} P^{*}}{\mathrm{~d} P} & =\exp \left\{-\frac{1}{2} \int_{0}^{T}\left(\sigma-\omega \pi_{t}\right)^{2} \mathrm{~d} t+\int_{0}^{T}\left(\sigma-\omega \pi_{t}\right) \mathrm{d} \bar{W}_{t}\right\} \\
& =\exp \left\{\frac{1}{2} \int_{0}^{T}\left(\sigma-\omega \pi_{t}\right)^{2} \mathrm{~d} t+\int_{0}^{T}\left(\sigma-\omega \pi_{t}\right) \mathrm{d} \tilde{W}_{t}\right\}
\end{aligned}
$$

where $\omega=\frac{a_{h}-a_{l}}{\sigma}$.

According to the Girsanov theorem, $\tilde{W}_{t}$ is a $P^{*}$ Brownian motion. Let $\pi_{t}=\frac{\Phi_{t}}{1+\Phi_{t}}$, we have

$$
\frac{\mathrm{d} \Phi_{t}}{\Phi_{t}}=\omega^{2} \pi_{t} \mathrm{~d} t+\omega \mathrm{d} \bar{W}_{t}
$$

Then, price process $X_{t}$ and process $\Phi_{t}$ satisfy the equations

$$
\left(\begin{array}{l}
\frac{\mathrm{d} X_{t}}{X_{t}} \\
\frac{\mathrm{d} \Phi_{t}}{\Phi_{t}}
\end{array}\right)=\left(\begin{array}{l}
a_{l}+\sigma \\
\omega \sigma
\end{array}\right) \mathrm{d} t+\left(\begin{array}{l}
\sigma \\
\omega
\end{array}\right) \mathrm{d} \tilde{W}_{t}
$$

So, $X$ and $\Phi$ are geometric Brownian motions under measure $P^{*}$. Moreover, $\sigma$-field generated by $\tilde{W}$ coincides with the one generated by $X$.

We define the likelihood process

$$
\eta_{t}=\exp \left\{-\frac{1}{2} \int_{0}^{t}\left(\sigma-\omega \pi_{s}\right)^{2} \mathrm{~d} s+\int_{0}^{t}\left(\omega \pi_{s}-\sigma\right) \mathrm{d} \tilde{W}_{s}\right\}
$$


We know that $\left(\tilde{W}, \mathcal{F}^{X}\right)$ is a $P^{*}$-Brownian motion, so $\eta$ is an $\mathcal{F}^{X}$-martingale under measure $P^{*}$.

We have:

$$
\begin{aligned}
& \frac{\mathrm{d} X_{t}}{X_{t}}=\left(a_{l}+\sigma^{2}\right) \mathrm{d} t+\sigma \mathrm{d} \tilde{W}_{t} \\
\Rightarrow & X_{t}=X_{0} \exp \left\{\int_{0}^{t}\left(a_{l}+\frac{\sigma^{2}}{2} \mathrm{~d} s+\int_{0}^{t} \sigma \mathrm{d} \tilde{W}_{t}\right)\right\} \\
\Rightarrow & \eta_{t} X_{t}=X_{0} \exp \left\{\int_{0}^{t}\left(a_{l}-\frac{1}{2} \omega^{2} \pi_{s}^{2}+\sigma \omega_{s}\right) \mathrm{d} s+\int_{0}^{t} \omega \pi_{s} \mathrm{~d} \tilde{W}_{s}\right\} \\
& \frac{\mathrm{d} \Phi_{t}}{\Phi_{t}}=\omega \sigma \mathrm{d} t+\omega \mathrm{d} \tilde{W}_{t} \\
\Rightarrow & \Phi_{t}=\Phi_{0} \exp \left\{\int_{0}^{t}\left(\omega \sigma-\frac{\omega^{2}}{2}\right) \mathrm{d} s+\int_{0}^{t} \sigma \mathrm{d} \tilde{W}_{t}\right\}
\end{aligned}
$$

Denote that $E^{*}$ is an expectation operator with respect to measure $P^{*}$ and let $\tau \leq T$ is an $\mathcal{F}^{X}$-stopping time. Then, by the property $\mathcal{F}^{X}$-martingale under measure $P^{*}$ of $\eta$ (see [4], theorem 11), we have:

$$
\begin{aligned}
& E\left[\mathrm{e}^{-\tau r} \cdot X_{\tau}\right]=E^{*}\left[\mathrm{e}^{-\tau r} \cdot \eta_{T} X_{\tau}\right] \\
= & E^{*}\left\{\mathrm{e}^{-\tau r} \cdot \eta_{\tau} X_{\tau}\right\}=\frac{X_{0}}{1+\Phi_{0}} E^{*}\left[\mathrm{e}^{\left(a_{l}-r\right) \tau}\left(1+\Phi_{\tau}\right)\right]
\end{aligned}
$$

Lemma 1. $X_{t}$ can be written as:

$$
X_{t}=X_{0} \mathrm{e}^{\varepsilon t} \cdot\left(\frac{\Phi_{t}}{\Phi_{0}}\right)^{\alpha}
$$

where: $\alpha=\frac{\sigma}{\omega}=\frac{\sigma^{2}}{a_{h}-a_{l}}$ and $\varepsilon=\frac{a_{h}+a_{l}-\sigma^{2}}{2}$

\section{Proof.}

We have:

$$
\begin{aligned}
X_{t} & =X_{0} \exp \left\{\int_{0}^{t}\left(a_{l}+\frac{\sigma^{2}}{2}\right) \mathrm{d} s+\int_{0}^{t} \sigma \mathrm{d} \tilde{\mathrm{W}}_{s}\right\} \\
& =X_{0} \mathrm{e}^{t\left(a_{l}+a_{h}-\sigma^{2}\right) / 2} \cdot \exp \left\{\int_{0}^{t}\left(\frac{a_{l}-a_{h}}{2}+\sigma^{2}\right) \mathrm{d} s+\int_{0}^{t} \sigma \mathrm{d} \tilde{\mathrm{W}}_{s}\right\} \\
& =X_{0} \mathrm{e}^{\varepsilon t} \cdot \exp \left\{\int_{0}^{t}\left(\frac{a_{l}-a_{h}}{2}+\sigma^{2}\right) \mathrm{d} s+\int_{0}^{t} \sigma \mathrm{d} \tilde{\mathrm{W}}_{s}\right\}
\end{aligned}
$$

and

$$
\begin{aligned}
\Phi_{t} & =\Phi_{0} \exp \left\{\int_{0}^{t}\left(\omega \sigma-\frac{\omega^{2}}{2}\right) \mathrm{d} s+\int_{0}^{t} \omega \mathrm{d} \tilde{\mathrm{W}}_{s}\right\} \\
& =\Phi_{0} \exp \left\{\frac{\omega}{2}\left[\int_{0}^{t}\left(\sigma^{2}-\frac{\omega \sigma}{2}\right) \mathrm{d} s+\int_{0}^{t} \sigma \mathrm{d} \tilde{\mathrm{W}}_{s}\right]\right\} \Rightarrow\left(\frac{\Phi_{t}}{\Phi_{0}}\right)^{\frac{\sigma}{\omega}} \\
& =\exp \left\{\int_{0}^{t}\left(\frac{a_{l}-a_{h}}{2}+\sigma^{2}\right) \mathrm{d} s+\int_{0}^{t} \sigma \mathrm{d} \tilde{\mathrm{W}}_{s}\right\} .
\end{aligned}
$$

We consider an optimal stopping problem as:

$$
\Gamma(t, y)=\sup _{0 \leq \tau \leq T-t} E^{*}\left[\mathrm{e}^{\left(a_{l}-r\right) \tau}\left(1+Y_{\tau}\right)\right]
$$

where:

$$
Y_{u}=y \exp \left\{\left(\sigma \omega-\frac{\omega^{2}}{2}\right) u+\omega \tilde{W}_{u}\right\}, u \geq 0
$$

where supremum is taken in $\mathcal{F}^{X}$-stopping time $\tau, 0 \leq \tau \leq T-t$ with respect to filtration generated by $\tilde{W}$. It can be seen that the optimal stopping time in (2.7) can be turned to the optimal stopping time in the problem (2.2).

Now, we study the optimal stopping problem (2.7). We will prove that existing an increasing and continuous monotone function:

$$
b:[0, T] \rightarrow[0, \infty)
$$

such that the stopping time

$$
\tau_{t, y}^{*}:=\inf \left\{u \in[0, T-t]: Y_{u} \leq b(t+u)\right\} \wedge(T-t)
$$

is an optimal stopping time for the problem (2.7).

$Y_{u}$ satisfies the equation:

$$
\frac{\mathrm{d} Y_{u}}{Y_{u}}=\left(a_{h}-a_{l}\right) \mathrm{d} u+\omega \mathrm{d} W_{u}, \quad u \geq 0
$$

On the other hand, we can write $Y_{u}=y Z_{u}$, where

$$
Z_{u}=\exp \left\{\left(\sigma u-\frac{\omega^{2}}{2}\right) u+u \mathrm{~W}_{u}\right\} .
$$

With this notation, we have:

$$
\Gamma(t, y)=\sup _{0 \leq \tau \leq T-t} E^{*}\left[\mathrm{e}^{\left(a_{l}-r\right) \tau}(1+y Z \tau)\right]
$$

Give $\tau=0$ in (2.7), we have

$$
\Gamma(t, y) \geq G(y):=E^{*}\left(1+Y_{0}\right)=E^{*}(1+y)=1+y .
$$

Define the continuation region $C$ :

$$
C=\{(t, y) \in[0, T] \times(0, \infty): \Gamma(t, y)>G(y)\}
$$

and the stopping region $D$ :

$$
D=\{(t, y) \in[0, T] \times(0, \infty): \Gamma(t, y)=G(y)\}
$$

According to general theory about optimal stopping problems, the stopping time

$$
\tau_{D}=\inf \left\{0 \leq s \leq T-t:\left(t+s, y_{s}\right) \in D\right\}
$$

is optimal stopping time problem (2.7). Thus, determining the optimal stopping time is sufficient to defining the stopping region $D$.

Theorem 1. There exists a right continuous and nondecreasing function 


$$
b:[0, T) \rightarrow\left[0, \frac{r-a_{l}}{a_{h}-r}\right]
$$

such that

$$
C=\{(t, y) \in[0, T) \times(0, \infty): y>b(t)\}
$$

Furthermore, supremum in (7) is achieved with the stopping time

$$
\tau_{D}=\inf \left\{0 \leq u \leq T-t: Y_{u} \leq b(t+u)\right\}
$$

\section{Proof.}

We know that

$$
\begin{aligned}
C & =\{(t, y): \Gamma(t, y)>G(y)\} \\
& =\{(t, y): \Gamma(t, y)>1+y\}
\end{aligned}
$$

with every fix $t \in[0, T)$ and $y^{\prime}>y>0$, assume that $(t, y) \in C$, there exists a stopping time $\tau$ such that:

$$
E^{*}\left[\mathrm{e}^{\left(a_{l}-r\right) \tau}\left(1+y Z_{\tau}\right)\right]>1+y
$$

So:

$$
\begin{aligned}
\Gamma\left(t, y^{\prime}\right) \geq & E^{*}\left[\mathrm{e}^{\left(a_{l}-r\right) \tau}\left(1+y^{\prime} Z_{\tau}\right)\right] \\
= & E^{*}\left[\mathrm{e}^{\left(a_{l}-r\right) \tau}\left(1+y Z_{\tau}\right)\right] \\
& +\left(y^{\prime}-y\right) E^{*}\left[\mathrm{e}^{(a l-r) \tau} Z_{\tau}\right] \\
> & 1+y+\left(y^{\prime}-y\right) E^{*}\left[e^{\left(a_{l}-r\right) \tau} Z_{\tau}\right]
\end{aligned}
$$

And process $H_{t}=\mathrm{e}^{\left(a_{l}-r\right) t} Z_{t}$ is a submartingale, so we have:

$$
\begin{aligned}
\Gamma\left(t, y^{\prime}\right) & >1+y+\left(y^{\prime}-y\right) E^{*}\left[\mathrm{e}^{\left(a_{l}-r\right) \cdot 0} Z_{0}\right] \\
& =1+y+y^{\prime}-y=1+y^{\prime}
\end{aligned}
$$

Therefore, $\left(t, y^{\prime}\right) \in C$. This proves that remaining a function $b:[0, T) \rightarrow[0, \infty)$

such that: $\quad C=\{(t, y): t \in[0, T): y>b(t)\}$

We have $\mathrm{e}^{\left(a_{l}-r\right) t}\left(1+Y_{t}\right)$ to be a submartingale for $t \leq \inf \left\{s: Y_{s} \leq \frac{r-a_{l}}{a_{h}-r}\right\}$, so all points in region $\left\{(t, z): z>\frac{r-a_{l}}{a_{h}-r}\right\}$ belong to the continuation region.

Therefore, $b(t) \leq \frac{r-a_{l}}{a_{h}-r}$. The monotonicity of $\mathrm{b}$ follows from monotonicity of function $t \rightarrow \Gamma(t, y)$.

The right continuity of $b(t)$ follows from the continuation region $C$ is an open set.

Theorem 2. Assume that $b$ is the function described above whose existence is proved in Lemma 1. Define stopping time:

$$
\tau^{*}=\inf \left\{t: X_{t} \leq \frac{X_{0}}{\Phi_{0}^{\alpha}} \mathrm{e}^{\varepsilon t} \cdot b^{\alpha}(t)\right\} \wedge T
$$

Then, $\tau^{*}$ attains the supremum in (2.2).

Proof. Deduce directly from Theorem 1 and Lemma 1 by replacing $Y_{t}$ by $\Phi_{t}$.

Theorem 3. The optimal stopping boundary $b(t)$ satisfies the integral equation (see Equation (2.9) below):

where $\Phi(x)=\frac{1}{\sqrt{2 \pi}} \int_{-\infty}^{x} \mathrm{e}^{-y^{2} / 2} \mathrm{~d} y$.

Proof.

Fix $t \in[0, T]$ and $Y_{0}=y \in(0, \infty)$. Then,

$$
\begin{aligned}
& \mathrm{e}^{\left(a_{l}-r\right)(T-t)} E^{*}\left[G\left(Y_{T-t}\right)\right] \\
= & \mathrm{e}^{\left(a_{l}-r\right)(T-t)} E^{*}\left[\Gamma\left(T, Y_{T-t}\right)\right]
\end{aligned}
$$

where $G(y)=1+y$.

But:

$$
\begin{aligned}
& \mathrm{e}^{\left(a_{l}-r\right)(T-t)} E^{*}\left[G\left(Y_{T-t}\right)\right] \\
= & E^{*}\left[\mathrm{e}^{\left(a_{l}-r\right)(T-t)}\right]+E^{*}\left[\mathrm{e}^{\left(a_{l}-r\right)(T-t)} Y_{T-t}\right] \\
= & \mathrm{e}^{\left(a_{l}-r\right)(T-t)}+y \cdot \mathrm{e}^{\left(a_{h}-r\right)(T-t)}
\end{aligned}
$$

Consider:

$$
\begin{aligned}
& \mathrm{e}^{\left(a_{l}-r\right)(T-t)} E^{*}\left[\Gamma\left(T, Y_{T-t}\right)\right] \\
= & \mathrm{e}^{\left(a_{l}-r\right) u} y\left(a_{h}-r\right) E^{*}\left[Z_{u} I\left(Y_{u} \leq b(t+u)\right)\right] \\
= & \mathrm{e}^{\left(a_{l}-r\right) u}\left(a_{l}-r\right) E^{*}\left[I\left(Y_{u} \leq b(t+u)\right)\right]
\end{aligned}
$$

We have:

$$
\begin{aligned}
1+b(t)= & \mathrm{e}^{\left(a_{l}-r\right)(T-t)}+b(t) \cdot \mathrm{e}^{\left(a_{h}-r\right)(T-t)}-\int_{0}^{T-t}\left\{\left(a_{l}-r\right) \mathrm{e}^{\left(a_{l}-r\right) u} \Phi\left(\frac{1}{\omega \sqrt{u}}\left[\ln \frac{(t+u)}{b(t)}-\omega \sigma u+\frac{\omega^{2} u}{2}\right]\right)\right. \\
& \left.+b(t)\left(a_{h}-r\right) \mathrm{e}^{\left(a_{h}-r\right) u} \Phi\left(\frac{1}{\omega \sqrt{u}}\left[\ln \frac{b(t+u)}{b(t)}-\omega \sigma u-\frac{\omega^{2} u}{2}\right]\right)\right\} \mathrm{d} u
\end{aligned}
$$




$$
\begin{aligned}
& E_{t, y}^{*}\left[Z_{u} I\left(Y_{u} \leq b(t+u)\right)\right] \\
= & E_{t, y}^{*}\left[\exp \left(\sigma \omega-\frac{\omega^{2}}{2}\right) u+\omega \mathrm{W}_{u}\right] \\
& \times I\left(\frac{\mathrm{W}_{u}}{\sqrt{u}} \leq \frac{\left.\ln \left(\frac{b(t+u)}{y}\right)+\left(\frac{\omega^{2}}{2}-\sigma \omega\right) u\right)}{\omega \sqrt{u}}\right) \\
= & \mathrm{e}^{\left(\sigma \omega-\frac{\omega^{2}}{2}\right) u} \cdot \int_{-\infty}^{d} \mathrm{e}^{\omega z} \cdot \frac{1}{\sqrt{2 \pi}} \cdot \mathrm{e}^{-\frac{z^{2}}{2 u}} \mathrm{~d} z=\mathrm{e}^{\left(\sigma \omega-\frac{\omega^{2}}{2}\right) u} \\
& \cdot \int_{-\infty}^{d} \frac{1}{\sqrt{2 \pi}} \cdot \mathrm{e}^{-\frac{(z-\omega u)^{2}}{2 u}} \cdot \mathrm{e}^{\frac{\omega^{2} u}{2}} \mathrm{~d} z=\mathrm{e}^{\sigma \omega u} \int_{-\infty}^{d} \frac{1}{\sqrt{2 \pi}} \cdot \mathrm{e}^{-\frac{(z-\omega u)^{2}}{2 u}} \\
\text { where } & d=\frac{b(t+u)}{\ln \frac{b\left(\frac{\omega^{2}}{2}-\sigma \omega\right)}{{ }^{2}}+u} \cdot
\end{aligned}
$$

$$
\begin{aligned}
& \text { Let } x=\frac{z-\omega u}{\sqrt{u}} \text { and } \\
& 1+b(i h)=\mathrm{e}^{\left(a_{l}-r\right)(n-i) h}+b(i h) \mathrm{e}^{\left(a_{h}-r\right)(n-i) h}-h \sum_{k=1}^{n-i}\left[\left(a_{l}-r\right) \mathrm{e}^{\left(a_{l}-r\right) k h} \Phi\left(\frac{1}{\omega \sqrt{k h}}\left(\ln \frac{b(i h+k h)}{b(i h)}-\omega \sigma k h+\frac{\omega^{2} k h}{2}\right)\right)\right] \\
& -h \sum_{k=1}^{n-i}\left[b(i h)\left(a_{h}-r\right) \mathrm{e}^{\left(a_{h}-r\right) k h} \Phi\left(\frac{1}{\omega \sqrt{k h}}\left(\ln \frac{b(i h+k h)}{b(i h)}-\omega \sigma k h-\frac{\omega^{2} k h}{2}\right)\right)\right]
\end{aligned}
$$

For $i=n-1$, we have equation

$$
\begin{aligned}
1+b\left(t_{n-1}\right)= & \mathrm{e}^{\left(a_{l}-r\right) h}+b\left(t_{n-1}\right) \mathrm{e}^{\left(a_{h}-r\right) h}-h\left(a_{l}-r\right) \mathrm{e}^{\left(a_{l}-r\right) h} \Phi\left(\frac{1}{\omega \sqrt{h}}\left(\ln \frac{b(T)}{b\left(t_{n-1}\right)}-\omega \sigma h+\frac{\omega^{2} h}{2}\right)\right) \\
& -h b\left(t_{n-1}\right)\left(a_{h}-r\right) \mathrm{e}^{\left(a_{h}-r\right) h} \Phi\left(\frac{1}{\omega \sqrt{h}}\left(\ln \frac{b(T)}{b\left(t_{n-1}\right)}-\omega \sigma h-\frac{\omega^{2} h}{2}\right)\right)
\end{aligned}
$$

Due to $b(T) \approx \frac{r-a_{l}}{a_{h}-r}$, from the above equation we determine $b\left(t_{n-1}\right)$, continue to $i=n-2$, we obtain the following equation for determining $b\left(t_{n-2}\right)$ :

$$
\begin{aligned}
1+b\left(t_{n-2}\right)= & \mathrm{e}^{\left(a_{l}-r\right) 2 h}+b\left(t_{n-2}\right) \mathrm{e}^{\left(a_{h}-r\right) 2 h}-h\left(a_{l}-r\right) \mathrm{e}^{\left(a_{l}-r\right) 2 h} \Phi\left(\frac{1}{\omega \sqrt{2 h}}\left(\ln \frac{b(T)}{b\left(t_{n-2}\right)}-\omega \sigma 2 h+\frac{\omega^{2} 2 h}{2}\right)\right) \\
& -h b\left(t_{n-1}\right)\left(a_{h}-r\right) \mathrm{e}^{\left(a_{h}-r\right) 2 h} \Phi\left(\frac{1}{\omega \sqrt{2 h}}\left(\ln \frac{b(T)}{b\left(t_{n-2}\right)}-\omega \sigma 2 h-\frac{\omega^{2} 2 h}{2}\right)\right) \\
& -h\left(a_{l}-r\right) \mathrm{e}^{\left(a_{l}-r\right) h} \Phi\left(\frac{1}{\omega \sqrt{h}}\left(\ln \frac{b\left(t_{n-1}\right)}{b\left(t_{n-2}\right)}-\omega \sigma h+\frac{\omega^{2} h}{2}\right)\right) \\
& -h b\left(t_{n-1}\right)\left(a_{h}-r\right) \mathrm{e}^{\left(a_{h}-r\right) h} \Phi\left(\frac{1}{\omega \sqrt{h}}\left(\ln \frac{b\left(t_{n-1}\right)}{b\left(t_{n-2}\right)}-\omega \sigma h-\frac{\omega^{2} h}{2}\right)\right)
\end{aligned}
$$


Just do so until $i=0$, it has been determined $b\left(t_{0}\right)$. Thus, we obtain a sequence of values $b\left(t_{0}\right), b\left(t_{1}\right), \cdots, b\left(t_{n}\right)$ of $b(t)$ and approximate the optimal boundaries for the asset liquidation process.

We have the approximate solution of Equation (2.9) by a computer program written in Matlab software, then set the boundaries for the process $X(t)$ is

$B(t)=\frac{X_{0}}{\Phi_{0}^{\alpha}} \mathrm{e}^{\varepsilon t} \cdot b^{\alpha}(t)$. Then the optimal time to sell is the first time the line describes the process $B(t)$ lies below the line describes the process $X(t)$. These figures (Figures 4-19) and tables below (Tables 1-3) illustrates the stopping time in (2.8) and the solutions of Equation (2.9) in some cases.

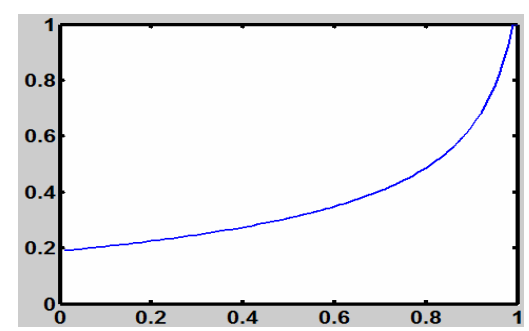

Figure 4. The line describes $b(t)$ with $a_{l}=0.1 ; a_{h}=0.2 ; r=$ $0.15 ; \sigma=0.2$.

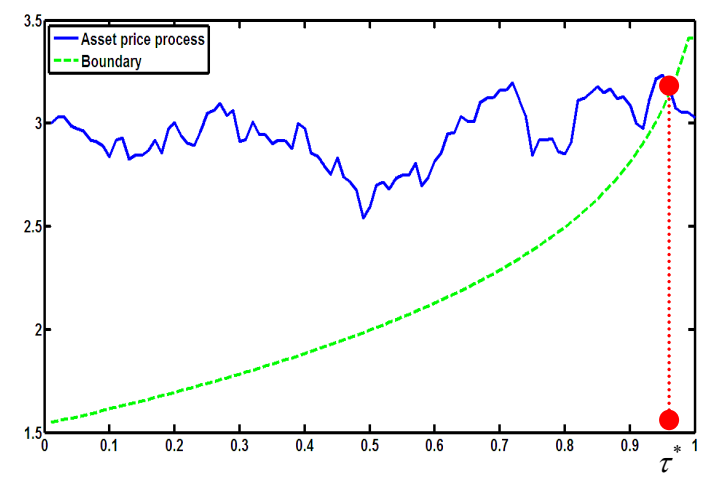

Figure 5. A simulation for the stopping time in (2.8) with parameters $X_{0}=3 ; a_{l}=0.1 ; a_{h}=0.2 ; r=0.15 ; \sigma=0.2 ; \pi_{0}=0.5$.

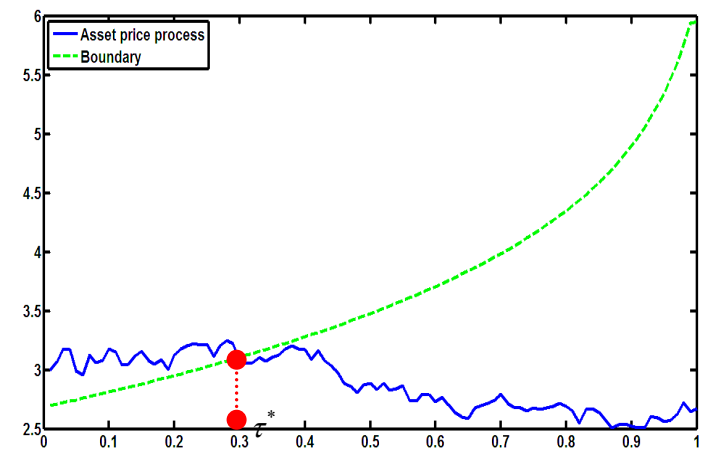

Figure 6. A simulation for the stopping time in (2.8) with parameters $X_{0}=3 ; a_{l}=0.1 ; a_{h}=0.2 ; r=0.15 ; \sigma=0.2 ; \pi_{0}=$ 0.2. In this case $\pi_{0}=0.2$ is small so is $\tau^{*}$.

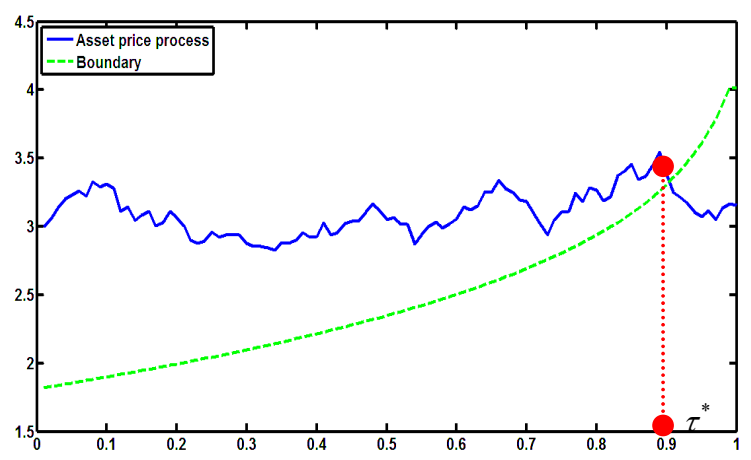

Figure 7. A simulation for the stopping time in (2.8) with parameters $X_{0}=3 ; a_{l}=0.1 ; a_{h}=0.2 ; r=0.15 ; \sigma=0.2 ; \pi_{0}=0.4$.

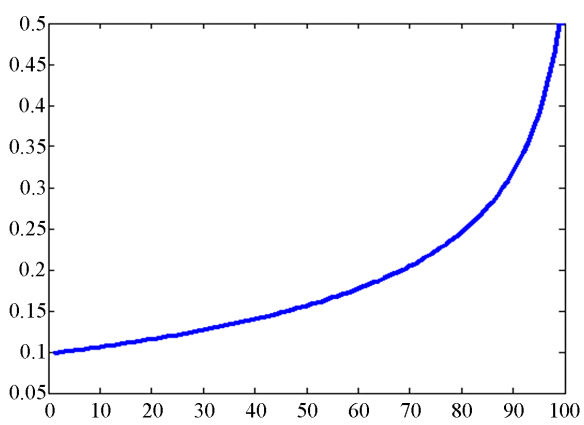

Figure 8. The line describes $b(t)$ with $a_{l}=0.09 ; a_{h}=0.15 ; r=$ $0.11 ; \sigma=0.1$.

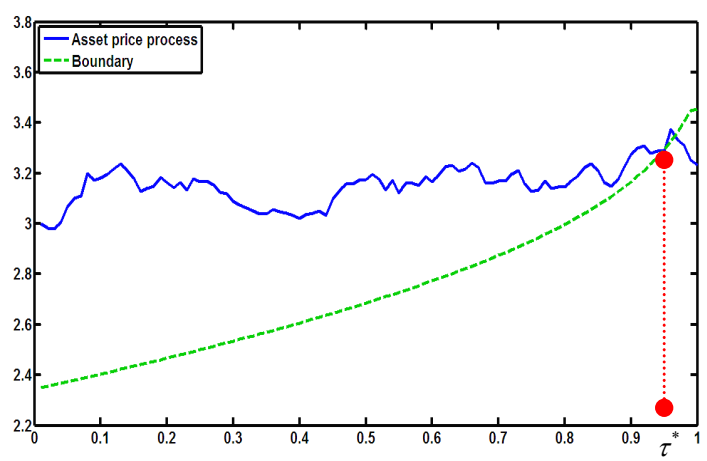

Figure 9. A simulation for the stopping time in (2.8) with parameters $X_{0}=3 ; a_{l}=0.09 ; a_{h}=0.15 ; r=0.11 ; \sigma=0.1 ; \pi_{0}=0.5$.

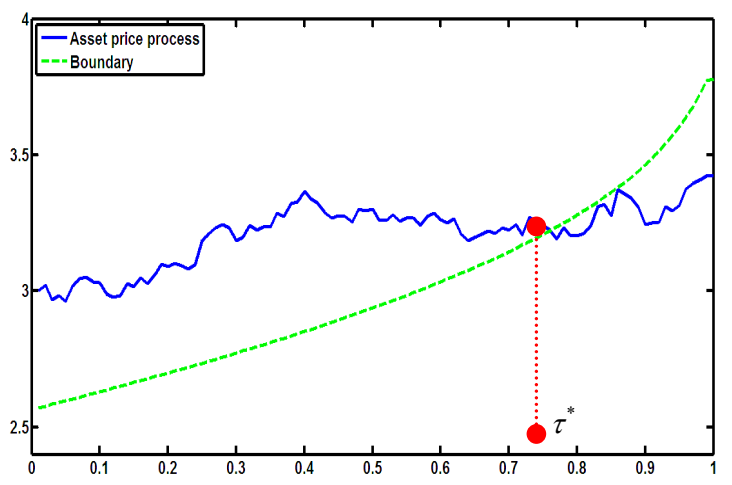

Figure 10. A simulation for the stopping time in (2.8) with parameters $X_{0}=3 ; a_{l}=0.09 ; a_{h}=0.15 ; r=0.11 ; \sigma=0.1 ; \pi_{0}=0.4$. 


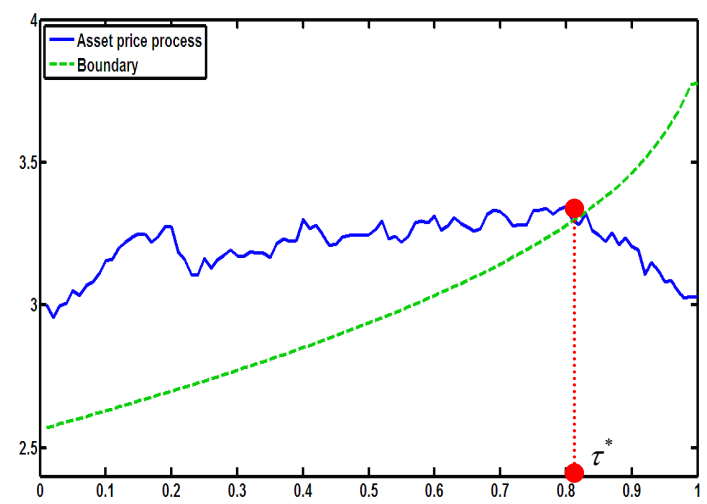

Figure 11. A simulation for the stopping time in (2.8) with parameters $X_{0}=3 ; a_{l}=0.09 ; a_{h}=0.15 ; r=0.11 ; \sigma=0.1 ; \pi_{0}=0.3$.

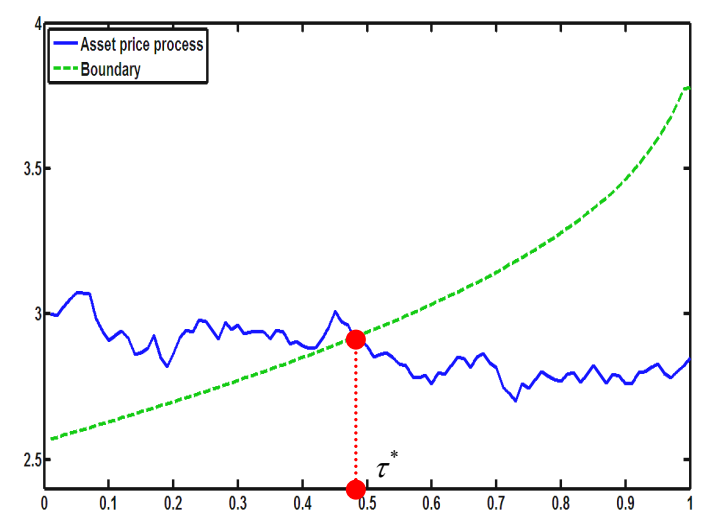

Figure 12. A simulation for the stopping time in (2.8) with parameters $X_{0}=3 ; a_{l}=0.09 ; a_{h}=0.15 ; r=0.11 ; \sigma=0.1 ; \pi_{0}=0.2$.

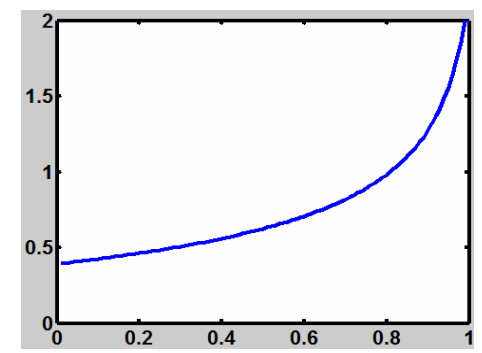

Figure 13. The line describes $b(t)$ with $a_{l}=0.09 ; a_{h}=0.15 ; r$ $=0.13 ; \sigma=0.1$.

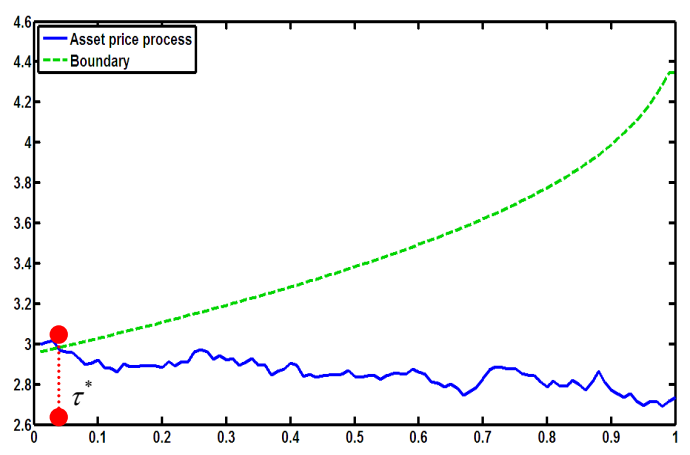

Figure 14. A simulation for the stopping time in (2.8) with parameters $X_{0}=3 ; a_{l}=0.09 ; a_{h}=0.15 ; r=0.13 ; \sigma=0.1 ; \pi_{0}=0.2$.

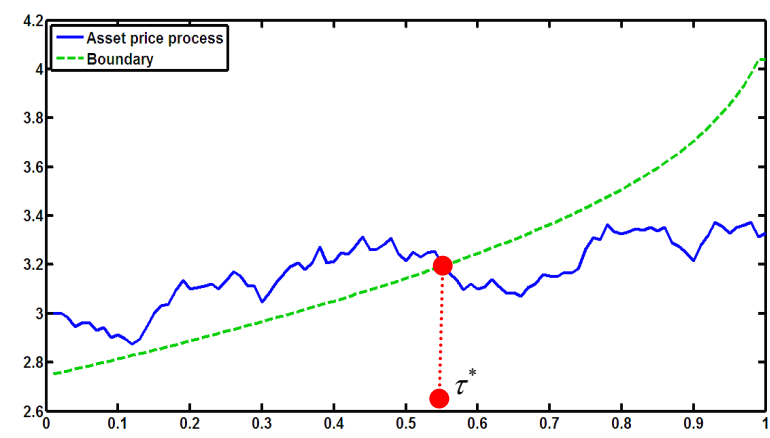

Figure 15. A simulation for the stopping time in (2.8) with parameters $X_{0}=3 ; a_{l}=0.09 ; a_{h}=0.15 ; r=0.13 ; \sigma=0.1 ; \pi_{0}=0.4$.

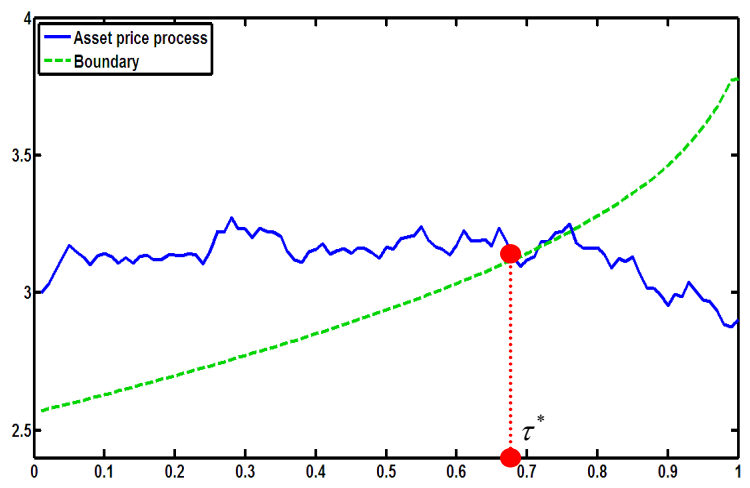

Figure 16. A simulation for the stopping time in (2.8) with parameters $X_{0}=3 ; a_{l}=0.09 ; a_{h}=0.15 ; r=0.13 ; \sigma=0.1 ; \pi_{0}=0.5$.

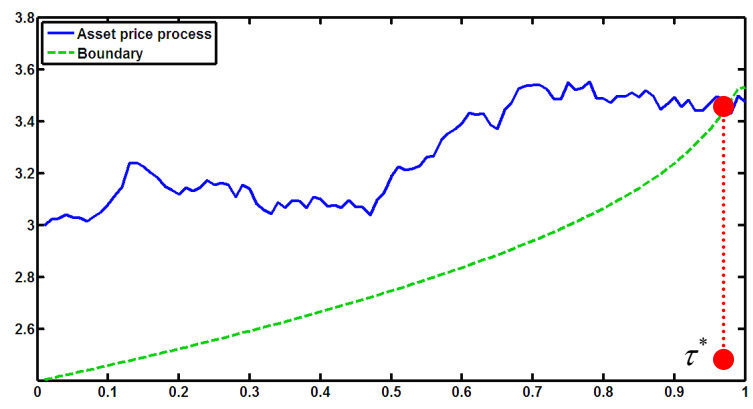

Figure 17. A simulation for the stopping time in (2.8) with parameters $X_{0}=3 ; a_{l}=0.09 ; a_{h}=0.15 ; r=0.13 ; \sigma=0.1 ; \pi_{0}=0.5$.

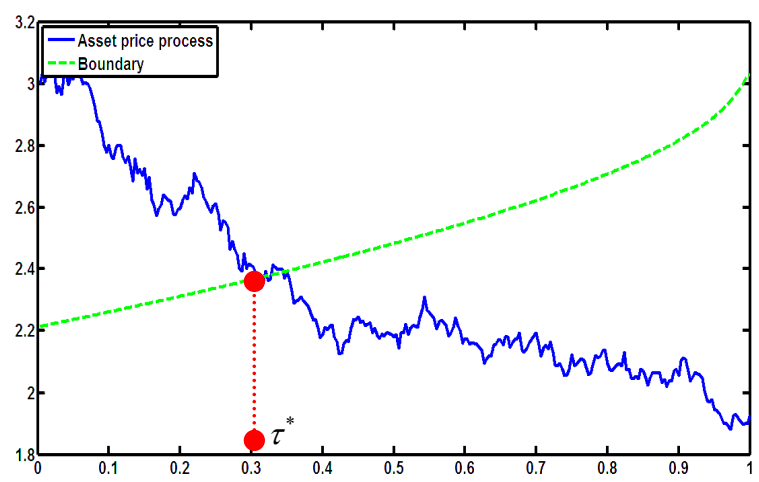

Figure 18. A simulation for the stopping time in (2.8) with parameters $X_{0}=3 ; a_{l}=-0.3 ; a_{h}=0.5 ; r=0.1 ; \sigma=0.2 ; \pi_{0}=0.4$. 


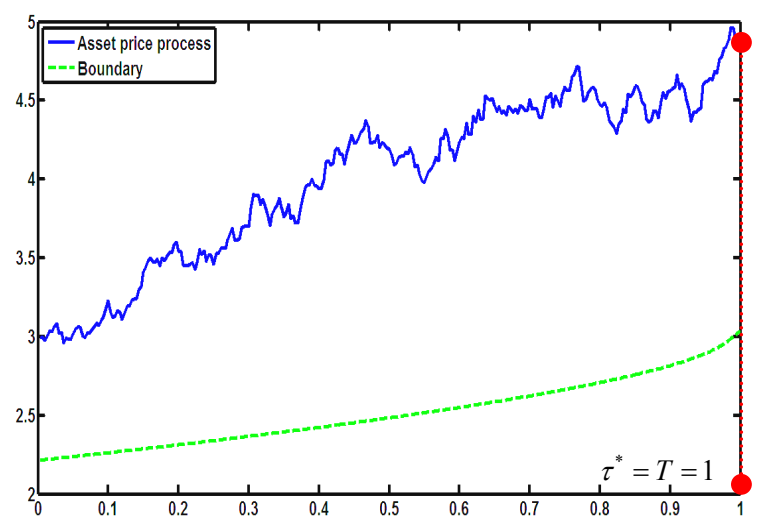

Figure 19. A simulation for the stopping time in (2.9) with parameters $X_{0}=3 ; a_{l}=-0.3 ; a_{h}=0.5 ; r=0.1 ; \sigma=0.2 ; \pi_{0}=0.6$.

Table 1. The numerical solutions of (2.9) with $a_{l}=0.1 ; a_{h}=$ $0.2 ; r=0.15 ; \sigma=0.2$.

\begin{tabular}{cccccccccc}
\hline$i$ & $\mathrm{~b}\left(t_{i}\right)$ & $i$ & $\mathrm{~b}\left(t_{i}\right)$ & $i$ & $\mathrm{~b}\left(t_{i}\right)$ & $i$ & $\mathrm{~b}\left(t_{i}\right)$ & $i$ & $\mathrm{~b}\left(t_{i}\right)$ \\
\hline 1 & 0.1909 & 21 & 0.2272 & 41 & 0.2775 & 61 & 0.3541 & 81 & 0.4985 \\
2 & 0.1924 & 22 & 0.2294 & 42 & 0.2806 & 62 & 0.359 & 82 & 0.5098 \\
3 & 0.1941 & 23 & 0.2315 & 43 & 0.2837 & 63 & 0.3642 & 83 & 0.5219 \\
4 & 0.1957 & 24 & 0.2337 & 44 & 0.2868 & 64 & 0.3695 & 84 & 0.5347 \\
5 & 0.1973 & 25 & 0.2359 & 45 & 0.2901 & 65 & 0.3749 & 85 & 0.5485 \\
6 & 0.199 & 26 & 0.2382 & 46 & 0.2934 & 66 & 0.3806 & 86 & 0.5632 \\
7 & 0.2007 & 27 & 0.2405 & 47 & 0.2968 & 67 & 0.3864 & 87 & 0.5791 \\
8 & 0.2024 & 28 & 0.2428 & 48 & 0.3003 & 68 & 0.3925 & 88 & 0.5963 \\
9 & 0.2042 & 29 & 0.2452 & 49 & 0.3038 & 69 & 0.3987 & 89 & 0.6151 \\
10 & 0.2059 & 30 & 0.2477 & 50 & 0.3074 & 70 & 0.4052 & 90 & 0.6356 \\
11 & 0.2077 & 31 & 0.2501 & 51 & 0.3112 & 71 & 0.412 & 91 & 0.6582 \\
12 & 0.2096 & 32 & 0.2526 & 52 & 0.315 & 72 & 0.419 & 92 & 0.6833 \\
13 & 0.2114 & 33 & 0.2552 & 53 & 0.3189 & 73 & 0.4263 & 93 & 0.7113 \\
14 & 0.2133 & 34 & 0.2578 & 54 & 0.3229 & 74 & 0.4339 & 94 & 0.743 \\
15 & 0.2152 & 35 & 0.2605 & 55 & 0.327 & 75 & 0.4419 & 95 & 0.7791 \\
16 & 0.2171 & 36 & 0.2632 & 56 & 0.3312 & 76 & 0.4502 & 96 & 0.8207 \\
17 & 0.2191 & 37 & 0.2659 & 57 & 0.3355 & 77 & 0.4589 & 97 & 0.8696 \\
18 & 0.2211 & 38 & 0.2687 & 58 & 0.34 & 78 & 0.468 & 98 & 0.9281 \\
19 & 0.2231 & 39 & 0.2716 & 59 & 0.3445 & 79 & 0.4777 & 99 & 0.9995 \\
20 & 0.2252 & 40 & 0.2745 & 60 & 0.3492 & 80 & 0.4878 & 100 & 1 \\
\hline
\end{tabular}

Table 2. The numerical solutions of (2.8) with $a_{l}=0.09 ; a_{h}=$ $0.15 ; r=0.11 ; \sigma=0.1$.

\begin{tabular}{cccccccccc}
\hline$i$ & $\mathrm{~b}\left(t_{i}\right)$ & $i$ & $\mathrm{~b}\left(t_{i}\right)$ & $i$ & $\mathrm{~b}\left(t_{i}\right)$ & $i$ & $\mathrm{~b}\left(t_{i}\right)$ & $i$ & $\mathrm{~b}\left(t_{i}\right)$ \\
\hline 1 & 0.0985 & 21 & 0.1165 & 41 & 0.1414 & 61 & 0.1792 & 81 & 0.2507 \\
2 & 0.0993 & 22 & 0.1176 & 42 & 0.1429 & 62 & 0.1817 & 82 & 0.2563 \\
3 & 0.1001 & 23 & 0.1186 & 43 & 0.1444 & 63 & 0.1842 & 83 & 0.2623 \\
4 & 0.1009 & 24 & 0.1197 & 44 & 0.146 & 64 & 0.1868 & 84 & 0.2686 \\
5 & 0.1017 & 25 & 0.1208 & 45 & 0.1476 & 65 & 0.1895 & 85 & 0.2754 \\
6 & 0.1025 & 26 & 0.122 & 46 & 0.1493 & 66 & 0.1923 & 86 & 0.2827 \\
7 & 0.1034 & 27 & 0.1231 & 47 & 0.1509 & 67 & 0.1952 & 87 & 0.2906 \\
8 & 0.1042 & 28 & 0.1243 & 48 & 0.1526 & 68 & 0.1982 & 88 & 0.2992 \\
9 & 0.1051 & 29 & 0.1254 & 49 & 0.1544 & 69 & 0.2013 & 89 & 0.3085 \\
10 & 0.106 & 30 & 0.1266 & 50 & 0.1562 & 70 & 0.2045 & 90 & 0.3186 \\
11 & 0.1069 & 31 & 0.1279 & 51 & 0.158 & 71 & 0.2079 & 91 & 0.3299 \\
12 & 0.1078 & 32 & 0.1291 & 52 & 0.1599 & 72 & 0.2113 & 92 & 0.3423 \\
\hline
\end{tabular}

\section{Continued}

\begin{tabular}{cccccccccc}
\hline 13 & 0.1087 & 33 & 0.1304 & 53 & 0.1618 & 73 & 0.2149 & 93 & 0.3563 \\
14 & 0.1096 & 34 & 0.1317 & 54 & 0.1638 & 74 & 0.2187 & 94 & 0.372 \\
15 & 0.1106 & 35 & 0.133 & 55 & 0.1658 & 75 & 0.2226 & 95 & 0.39 \\
16 & 0.1115 & 36 & 0.1343 & 56 & 0.1679 & 76 & 0.2268 & 96 & 0.4107 \\
17 & 0.1125 & 37 & 0.1357 & 57 & 0.1701 & 77 & 0.2311 & 97 & 0.4351 \\
18 & 0.1135 & 38 & 0.1371 & 58 & 0.1723 & 78 & 0.2356 & 98 & 0.4642 \\
19 & 0.1145 & 39 & 0.1385 & 59 & 0.1745 & 79 & 0.2403 & 99 & 0.4999 \\
20 & 0.1155 & 40 & 0.1399 & 60 & 0.1768 & 80 & 0.2454 & 100 & 0.5 \\
\hline
\end{tabular}

Table 3. The numerical solutions of (2.9) with $a_{l}=0.09 ; a_{h}=$ $0.15 ; r=0.13 ; \sigma=0.1$.

\begin{tabular}{cccccccccc}
\hline$i$ & $\mathrm{~b}\left(t_{i}\right)$ & $i$ & $\mathrm{~b}\left(t_{i}\right)$ & $i$ & $\mathrm{~b}\left(t_{i}\right)$ & $i$ & $\mathrm{~b}\left(t_{i}\right)$ & $i$ & $\mathrm{~b}\left(t_{i}\right)$ \\
\hline 1 & 0.3944 & 21 & 0.4664 & 41 & 0.5658 & 61 & 0.717 & 81 & 1.0027 \\
2 & 0.3975 & 22 & 0.4706 & 42 & 0.5718 & 62 & 0.7269 & 82 & 1.0252 \\
3 & 0.4007 & 23 & 0.4749 & 43 & 0.578 & 63 & 0.737 & 83 & 1.0491 \\
4 & 0.4039 & 24 & 0.4792 & 44 & 0.5843 & 64 & 0.7475 & 84 & 1.0745 \\
5 & 0.4072 & 25 & 0.4836 & 45 & 0.5907 & 65 & 0.7583 & 85 & 1.1018 \\
6 & 0.4105 & 26 & 0.4881 & 46 & 0.5972 & 66 & 0.7695 & 86 & 1.131 \\
7 & 0.4139 & 27 & 0.4927 & 47 & 0.6039 & 67 & 0.781 & 87 & 1.1625 \\
8 & 0.4173 & 28 & 0.4973 & 48 & 0.6108 & 68 & 0.793 & 88 & 1.1967 \\
9 & 0.4208 & 29 & 0.502 & 49 & 0.6178 & 69 & 0.8054 & 89 & 1.2339 \\
10 & 0.4243 & 30 & 0.5068 & 50 & 0.6249 & 70 & 0.8182 & 90 & 1.2746 \\
11 & 0.4278 & 31 & 0.5117 & 51 & 0.6323 & 71 & 0.8316 & 91 & 1.3195 \\
12 & 0.4314 & 32 & 0.5167 & 52 & 0.6398 & 72 & 0.8454 & 92 & 1.3694 \\
13 & 0.4351 & 33 & 0.5217 & 53 & 0.6475 & 73 & 0.8599 & 93 & 1.4252 \\
14 & 0.4388 & 34 & 0.5269 & 54 & 0.6554 & 74 & 0.8749 & 94 & 1.4881 \\
15 & 0.4426 & 35 & 0.5321 & 55 & 0.6635 & 75 & 0.8907 & 95 & 1.56 \\
16 & 0.4464 & 36 & 0.5375 & 56 & 0.6719 & 76 & 0.9071 & 96 & 1.6429 \\
17 & 0.4503 & 37 & 0.5429 & 57 & 0.6804 & 77 & 0.9244 & 97 & 1.7403 \\
18 & 0.4542 & 38 & 0.5485 & 58 & 0.6892 & 78 & 0.9424 & 98 & 1.857 \\
19 & 0.4582 & 39 & 0.5542 & 59 & 0.6982 & 79 & 0.9615 & 99 & 1.9994 \\
20 & 0.4623 & 40 & 0.5599 & 60 & 0.7075 & 80 & 0.9815 & 100 & 2 \\
\hline
\end{tabular}

\section{Conclusion}

This paper solves the problem to find the optimal stopping time for the holding asset and make a decision when to sell assets with discounted price reaching the greatest expected value. The optimal stopping time is the first time the price of the asset hit the boundary or be at the time $T$. In next study, we will study the distributions and characteristics of the optimal stopping time.

\section{REFERENCES}

[1] G. Peskir and A. N. Shiryaev, "Optimal Stopping and Free-Boundary Problems (Lectures in Mathematics ETH Lectures in Mathematics. ETH Zürich (Closed))," Birkhäuser, Basel, 2006.

[2] A. N. Shiryaev, Z. Xu and X. Y. Zhou, "Thou Shalt Buy and Hold," Quantitative Finance, Vol. 8, No. 8, 2008, pp. 765-776.

[3] R. S. Lipster and A. N. Shiryaev, "Statistics of Random Process: I. General Theory,” Springer-Verlag, Berlin, Heidelberg, 2001. 
[4] A. N. Shiryaev, "Optimal Stopping Rules," SpringerVerlag, Berlin, Heidelberg, 1978, 2008.

[5] X. L. Zhang, "Numerical Analysis of American Option
Pricing in a Jump-Diffusion Model," Mathematics of Operations Research, Vol. 22, No. 3, 1997, pp. 668-690. doi:10.1287/moor.22.3.668 